Aim of the study: The purpose of this study was to create and introduce a Polish version of the European Organisation for Research and Treatment of Cancer (EORTC) Quality of Life Questionnaire for Endometrial Cancer (QLQ-EN24).

Material and methods: The translation procedure described by the EORTC was adopted; this comprised two independent forward translations of the original English questionnaire into Polish, two independent backward translations from Polish to English and pilot testing conducted on 12 patients with diagnosed endometrial cancer. They were asked whether there was any difficulty in answering, confusion while answering, or difficulty in understanding the questions, and if the patients themselves would have framed the questions in a different way.

Results: During the process of translation, discussions and text corrections were performed by medical experts and native English speakers. Some sentences or phrasing were adopted from other EORTC questionnaires for different diseases. After pilot testing, further text corrections were adopted according to patients' comments. These involved wording, sentence structure, and text editing (font size, bolding).

Conclusions: The final version of the Polish EN24 questionnaire has been approved by the EORTC Translational Office. It is available on the EORTC web page now.

Key words: quality of life, question naire, endometrial cancer, translation, adaptation.

Contemp Oncol (Pozn) 2014; 18 (2): 134-139 DOI: $10.5114 /$ wo.2014.40621

\section{Creation of the Polish version and adaptation of the European Organisation for Research and Treatment of Cancer Quality of Life Questionnaire-Endometrial Cancer Module (QLQ-EN24)}

\author{
Maciej Stukan ${ }^{1}$, Kamil Zalewski ${ }^{2,3}$, Jacek P. Grabowski ${ }^{4,5}$, Marcin Mardas ${ }^{5}$, \\ Steven Jones ${ }^{6}$, Małgorzata Pietrzak-Stukan ${ }^{7}$, Mirosław Dudziak ${ }^{1}$
}

\author{
1Department of Gynecologic Oncology, Gdynia Oncology Center, Gdynia, Poland \\ 2Department of Clinical Oncology, Holycross Cancer Center, Kielce, Poland \\ ${ }^{3}$ Chair and Clinical Department of Obstetrics, Female Diseases and Gynecological \\ Oncology, II Medical Faculty, Medical University of Warsaw, Warsaw, Poland \\ ${ }^{4}$ Department of Gynecology and Gynecologic Oncology, Kliniken-Essen-Mitte, Essen, \\ Germany \\ Department of Oncology, Poznan University of Medical Sciences, Polska \\ ${ }^{6}$ Distinction Language Centre, Gdansk, Poland \\ 'Obstetric Department, Medical University of Gdańsk, Poland \\ Authors: M. Stukan, K. Zalewski, J.P. Grabowski, M. Mardas are members of European \\ Network of Young Gynaecologist Oncologists (ENYGO)
}

\section{Introduction}

Endometrial cancer is the most common pelvic gynecological malignancy in high-income countries, with a 2-3\% lifetime risk of developing the disease $[1,2]$. Most carcinomas are diagnosed at an early stage. Still, $15-20 \%$ of these recur as a metastatic disease. Patients with intermediate and high risk for recurrence are offered extensive surgery including pelvic and paraaortic lymphadenectomy, followed by adjuvant radiotherapy, chemotherapy, or both [1]. This treatment is associated with acute and delayed complications in elderly and multimorbid patients. Nevertheless, only 10-15\% of these women might benefit from such additional therapy [3]. Thus there is a need for improvement in the way patients are classified into high-risk of recurrence in order to tailor surgical treatment, propose effective adjuvant therapies and introduce new target treatment for selected groups of patients in whom disease is most likely to recur. As a consequence, the development of new clinical trials is necessary. Quality of life (QOL) is a very important issue in current oncology. In contemporary clinical trials, the QOL assessment is performed together with collection of disease-specific end points. The European Organisation for Research and Treatment of Cancer (EORTC) is a professional, international institution, including a specialized Group for Quality of Life. Many questionnaires were developed and are available for different malignancies. The original, specific questionnaire for endometrial cancer was named EN24 [4]. The aim of our study was to create a Polish version of the EORTC questionnaire for quality of life assessment in patients with endometrial cancer (EN24).

\section{Material and methods}

EORTC Quality of Life Questionnaire for Endometrial Cancer, English version of module EN24 (http://groups.eortc.be/qol/eortc-modules) was taken as a basis to create the Polish version [4]. The procedure was consistent with translation guidelines [5]. The EORTC Translation Office first presented 
the English original version together with an EN24 Polish interim version, where some translations were adopted from a similar module for cervical cancer, CX24 [6]. Two independent forward translations (from English to Polish), together with corrections of the interim version, were performed by medical experts. The first Polish version was created. Later two independent backward translations (from Polish to English) were performed by native English speaking translators. To some extent, translation issues were discussed via e-mail, as well as direct meetings and teleconferences. Afterwards the Polish version together with the backward translation into English were presented to the EORTC Translation Office.

After the translation, pilot testing was performed to identify and solve any potential problems in the translation, including vocabulary issues and expressions that would be most suitable for the target population. Pilot testing of the Polish version was conducted on 12 patients with endometrial cancer, admitted to the Department of Gynecologic Oncology Gdynia Oncology Center between August and October 2011. Bioethical Committee approval has been granted for this project. All patients participating in the study signed a consent form before completing the questionnaire. The initial EN24 questionnaire translation was presented and after completion a structured interview with each patient was conducted to determine whether the wording presented any difficulties in answering or understanding, whether the questions were confusing, upsetting or offensive, or whether the patient would have asked the question in a different way. According to patients' comments, the final Polish EN24 questionnaire version was created and presented to the EORTC Translational Office.

\section{Results}

The summary of the translation process is presented in Table 1, abbreviated to the most important linguistic controversies and solutions undertaken. In those cases where we used an available translation from another EORTC questionnaire, we did not present it in the table, though the issue was discussed for the best version. The translated and accepted first Polish version of the EORTC questionnaire EN24 was presented to 12 women with diagnosed endometrial cancer. The mean time to answer the EN24 module was 6 minutes (range 3-12 minutes) and the interview took from 1 to 10 minutes. The mean age of patients was 69 years (range 54 81 years). Polish was their native language and they lived in northern Poland. Eight of the women were retired. All patients were diagnosed based on histology examination from endometrial biopsy. In cases where metastatic disease was suspected, additional magnetic resonance imaging or computed tomography scans were performed. The cancer stages were from IA to IIIC in the studied group based on findings from staging surgery and imaging. The histological types of cancer were as follows: 9 patients with endometrioid, 2 with mixed endometrioid-serous, and one with serous adenocarcinoma. At the time of completing the questionnaire, 10 patients were about to undergo surgery, and 2 with contraindications to surgery were awaiting radical chemoradiotherapy.

\section{General linguistic considerations}

None of the interviewed women were sexually interested or active within the relevant period of time (4 weeks) and only two of them answered items number 51-54. Nevertheless, these questions were acceptable, understood, and not confusing or offensive. Individually interviewed patients recognized these items as important and well constructed.

One patient noted a discrepancy between the heading describing the time period and following questions. She suggested changing the heading and that the question style for all items be more uniform. This is because of the heading "During the last week" which was translated into Polish: "Czy w ostatnim tygodniu". The Polish "Czy" meaning depends on further words, and can be understood as: "Have you...", or "Did you...". When "Czy" is used in the heading, than there is no need to repeat it in every question. That is why we decided to erase word "Czy" from questions 39 and 40 of the first EN24 version.

The opposite problem occurred in the heading for items 49 and 50. Because of linguistic differences and the character of the following questions, the best form of the heading would be without "Czy" (present in the first version). It was decided to use "Podczas ostatnich czterech tygodni" (During the last four weeks) in the final version. Items 49 and 50 remain unchanged.

The heading for items 51-54 did not include the word "Czy", which is why we suggested adding this "prefix" to questions 51 and 52. "Czy" is used in a question sentence form, meaning: "Have you..." or "Did you...".

One patient proposed changing the word "Wcale" (Not at all) into the heading "Nie" (No). The suggestion was proposed after a problem with question 35 . The research team analyzed the problem, and decided to leave it as in the primary version with "Wcale" (Not at all), firstly because it is used in all EORTC Polish questionnaires, and secondly because we changed the problematic question 35 (see below).

\section{Detailed linguistic considerations \\ Question 33}

One patient suggested erasing the word "krzyża" meaning "back", referring to back pain. It was one opinion only, and the problem of pain in the lower back is different from and as important as pain in the pelvis, so the research team decided to leave the item unchanged.

\section{Question 35}

The form of question: "Oddawała Pani często mocz?" (Have you passed urine frequently?) and the answer: "Wcale" (Not at all) was confusing for two patients. This answer to the question could be understood as not passing urine at all. We proposed changing the question into: "Miała Pani problem z częstym oddawaniem moczu?", which was a better translation of the original English version, and did not cause any confusion.

Other questionnaire items were found to be acceptable and the patients had no comments.

All the patients' comments were taken into consideration and the final Polish EN24 questionnaire version was 


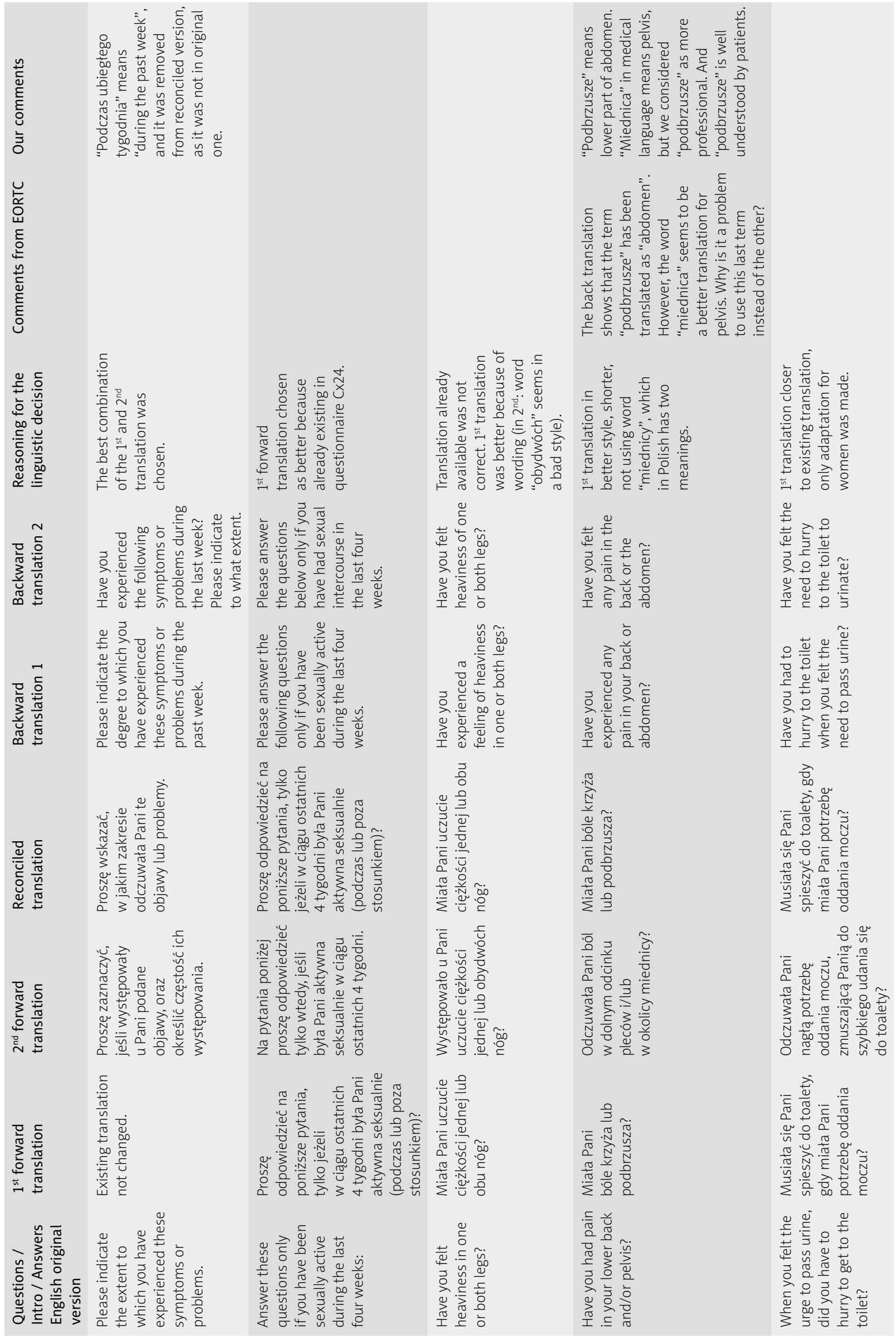




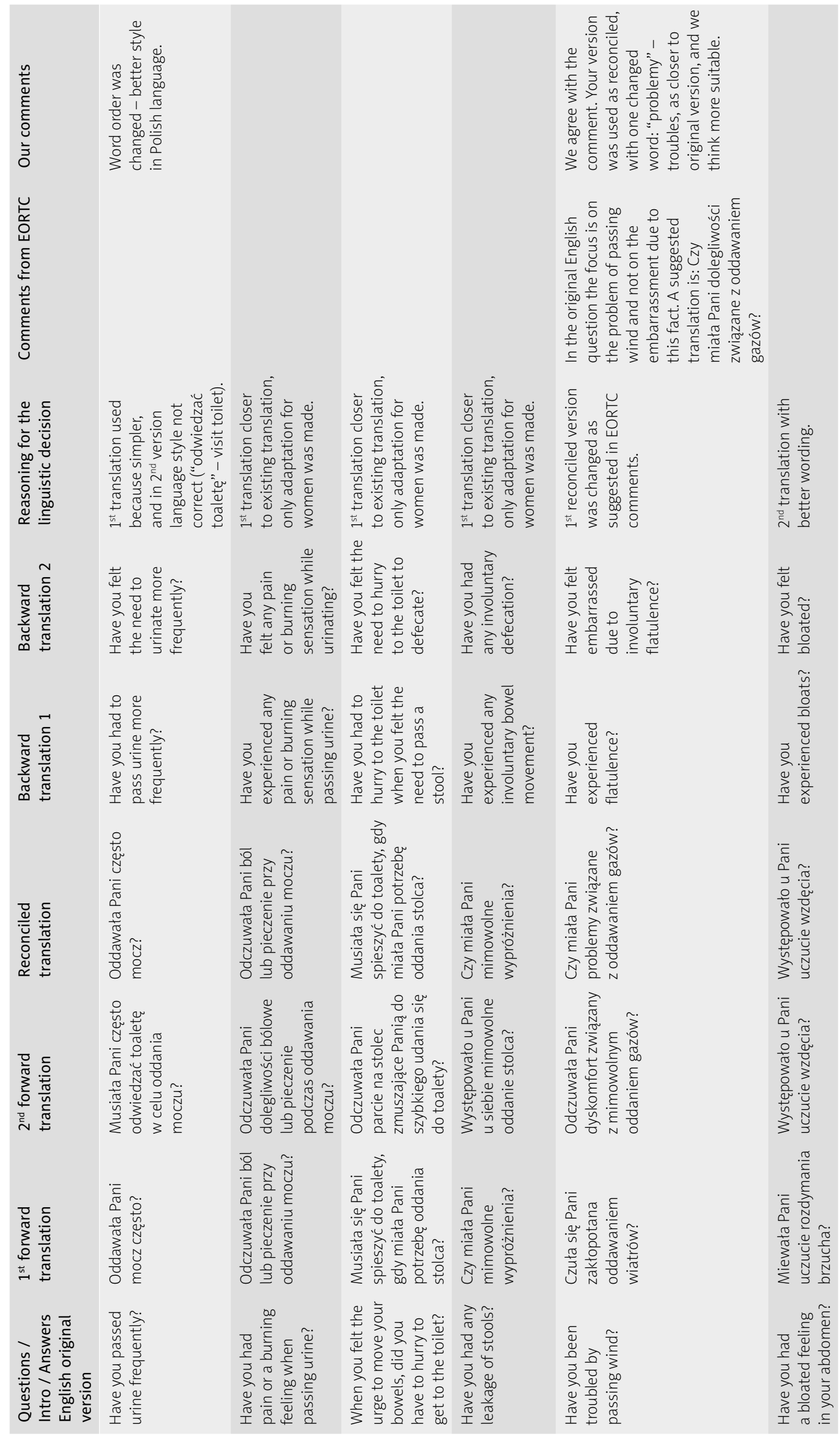




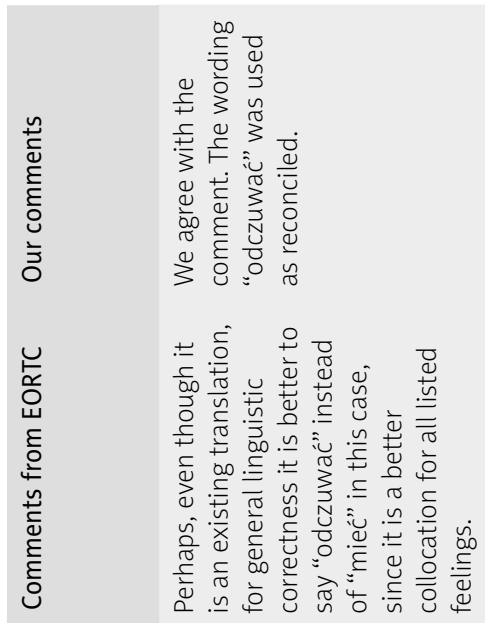

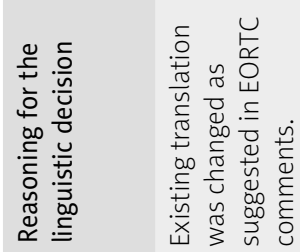
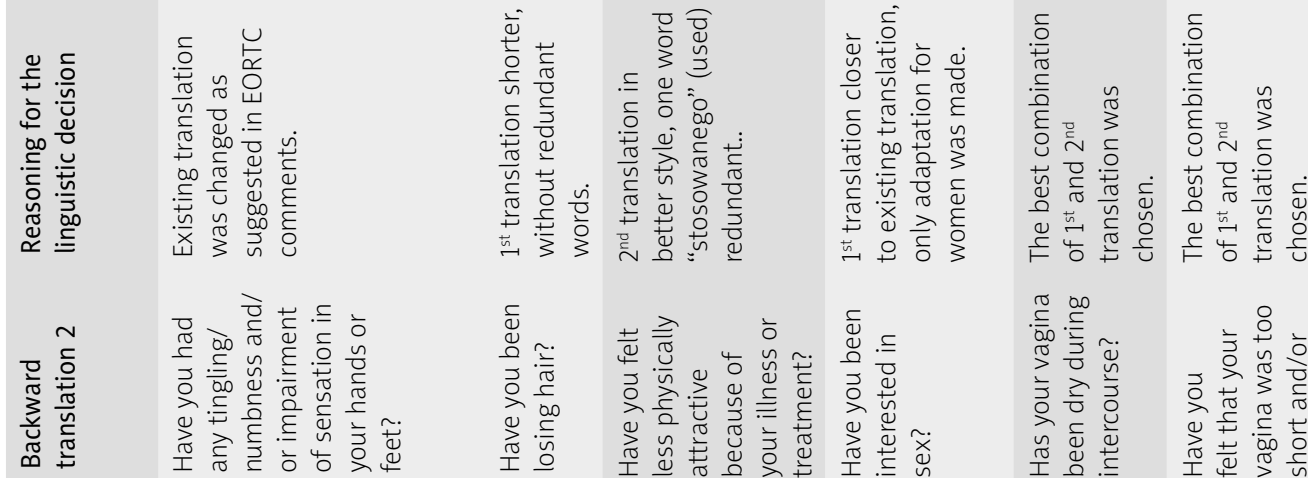

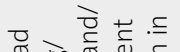

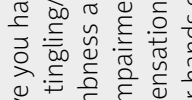

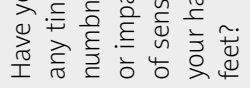

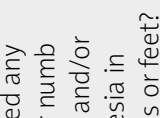

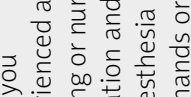

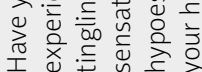
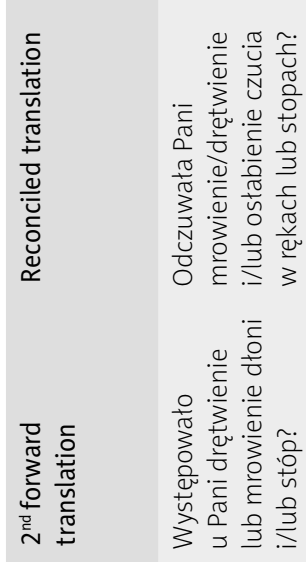

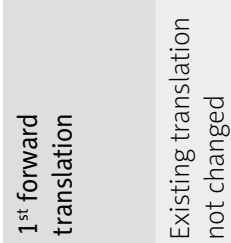

高

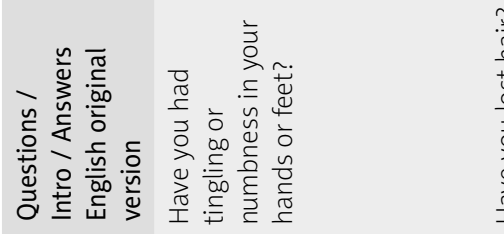

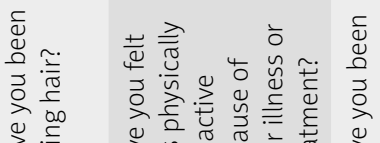

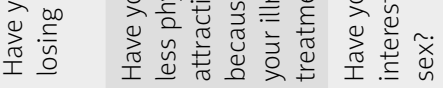

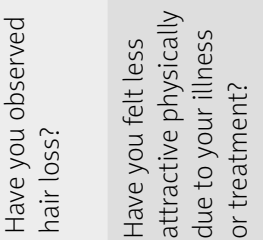

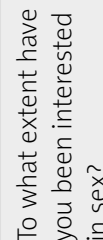

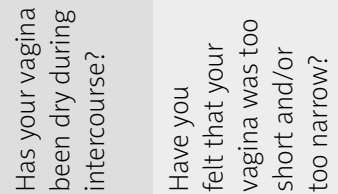

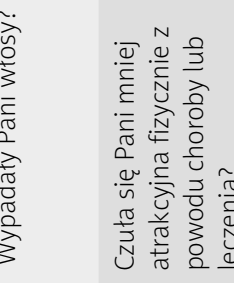

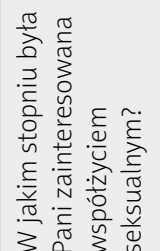

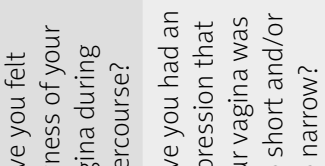

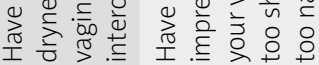

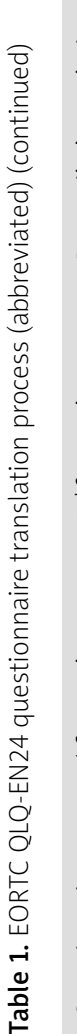

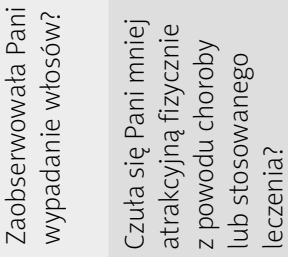

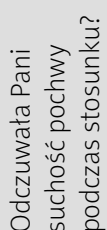

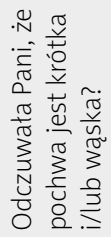

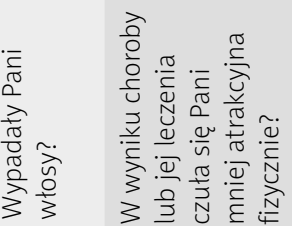

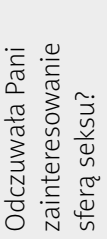

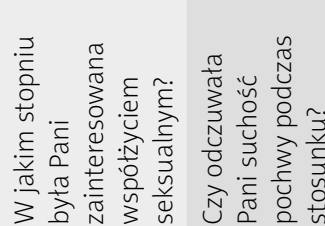

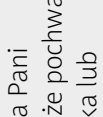

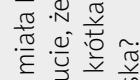

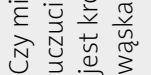

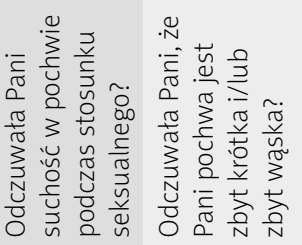

\begin{tabular}{|c|c|c|}
\hline 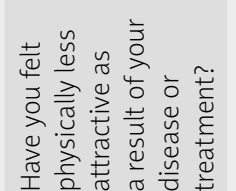 & 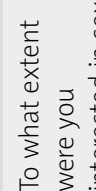 & 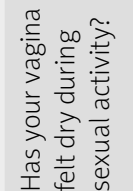 \\
\hline
\end{tabular}


created and presented to the EORTC Translation Office. This was accepted and is officially available on the EORTC Quality of Life Group web site (http://groups.eortc.be/qol/ eortc-modules).

\section{Discussion}

The QOL is an important issue in most contemporary oncological trials. Different malignancies cause different symptoms, both due to clinical presentation and treatment modality. The EORTC has developed special questionnaires for different diseases. These have been translated into many languages. The same tool for assessing the quality of life can be used in clinical trials conducted worldwide, or it is possible to compare QOL issues between trials where different treatment modalities within the same disease are examined. According to the guidelines, we translated and adapted the Polish version of the EN24, which was accepted by the EORTC Translation Office and is readily available on the EORTC web site (registration and permission required).

The questionnaire consists of simple questions to be answered by matching the most appropriate available answer or level of symptom intensity. The EN24 like other EORTC questionnaires is designed to be used by patients during all possible treatment modalities or disease phases. The questions seem to be positioned randomly on the sheet, but they are grouped and assessed together in separate scales (physical, social, emotional, etc.). After using the scoring system the results are presented in numbers, separately for each scale. The EN24 was constructed to match as an additional module to the general universal C30 questionnaire, and together these cover most quality of life assessment issues. Along with physical symptoms, other aspects are also addressed.

In specific patient subgroups or profiled clinical trials it can be more appropriate to use special, focused questionnaires, e.g. for depression (HADS), or sexual functioning (FSFI) [7-10]. The questionnaire EN24 has been used worldwide $[11,12]$. Researchers suggest that BMI should be considered and assessed additionally to questionnaires [13]. Another questionnaire specific for endometrial cancer is called FACT-En (Functional Assessment of Cancer Therapy Endometrial Cancer) (www.facit.org). It was used in one publication [14]. Well-known questionnaires such as the SF-36 or the shorter SF-12 are often used but there is no specific module for endometrial cancer (www.sf-36.org).

In conclusions the EORTC-EN24 is a simple tool for a quality of life assessment with appropriate wording and an easy to complete format. Used in clinical trials worldwide and having been tested on the Polish target population, it is now available for use in Poland. The validation of the EN24 questionnaire on larger groups of patients is now being performed by the authors.

Authors declare no conflict of interests.

The authors would like to acknowledge Elfriede Greimel and people from the EORTC Translation Office for their logistic support. I

\section{References}

1. Salvesen HB, Haldorsen IS, Trovik J. Markers for individualised therapy in endometrial carcinoma. Lancet Oncol 2012; 13: e353-61.

2. Amant F, Moerman P, Neven P, Timmerman D, Van Limbergen E, Vergote I. Endometrial cancer. Lancet 2005; 366: 491-505.

3. ASTEC/EN.5 Study Group, Blake P, Swart AM, Orton J, et al. Adjuvant external beam radiotherapy in the treatment of endometrial cancer (MRC ASTEC and NCIC CTG EN.5 randomised trials): pooled trial results, systematic review, and meta-analysis. Lancet 2009; 373: $137-46$.

4. Greimel E, Nordin A, Lanceley A, et al. Psychometric validation of the European Organisation for Research and Treatment of Cancer Quality of Life Questionnaire-Endometrial Cancer Module (EORTC QLQ-EN24). Eur J Cancer 2011; 47: 183-90.

5. Dewolf L, Koller M, Velikova G, Johnson C, Scott N, Bottomley A. A EORTC Quality of Life Group Translation Procedure. In: $3^{\text {rd }}$ edition EORTC Translation Procedure. Brussels 2009.

6. Greimel ER, Kuljanic Vlasic K, Waldenstrom AC, et al. The European Organization for Research and Treatment of Cancer (EORTC) Quality-of-Life questionnaire cervical cancer module: EORTC QLQCX24. Cancer 2006; 107: 1812-22.

7. Zigmond AS, Snaith RP. The hospital anxiety and depression scale. Acta Psychiatr Scand 1983; 67: 361-70.

8. Wiegel M, Meston C, Rosen R. The female sexual function index (FSFI): cross-validation and development of clinical cutoff scores. J Sex Marital Ther 2005; 31: 1-20.

9. Baser RE, Li Y, Carter J. Psychometric validation of the Female Sexual Function Index (FSFI) in cancer survivors. Cancer 2012; 11: 4606-18.

10. Nowosielski K, Wróbel B, Sioma-Markowska U, Poręba R. Development and validation of the Polish version of the Female Sexual Function Index in the Polish population of females. J Sex Med 2013; 10: 386-95.

11. van de Poll-Franse LV, Pijnenborg JM, Boll D, Vos MC, van den Berg $H$, Lybeert ML, de Winter K, Kruitwagen RF. Health related quality of life and symptoms after pelvic lymphadenectomy or radiotherapy vs. no adjuvant regional treatment in early-stage endometrial carcinoma: a large population-based study. Gynecol Oncol 2012; 127: 153-60.

12. Nicolaije KA, Ezendam NP, Vos MC, Boll D, Pijnenborg JM, Kruitwagen RF, Lybeert ML, van de Poll-Franse LV. Follow-up practice in endometrial cancer and the association with patient and hospital characteristics: a study from the population-based PROFILES registry. Gynecol Oncol 2013; 129: 324-31.

13. Oldenburg CS, Boll D, Nicolaije KA, et al. The relationship of body mass index with quality of life among endometrial cancer survivors: a study from the population-based PROFILES registry. Gynecol Oncol 2013; 129: 216-21.

14. Onujiogu N, Johnson T, Seo S, Mijal K, Rash J, Seaborne L, Rose S, Kushner DM. Survivors of endometrial cancer: who is at risk for sexual dysfunction? Gynecol Oncol 2011; 123: 356-9.

\section{Address for correspondence}

Maciej Stukan MD, PhD

Department of Gynecologic Oncology

Gdynia Oncology Center

Powstania Styczniowego 1

81-519 Gdynia, Poland

e-mail: maciej.stukan@gmail.com

Submitted: 29.05 .2013

Accepted: $\quad 16.07 .2013$ 